Conclusions Anxiety and depression among gastroenterologists are more prevalent in comparison to the general population. Gastroenterologist of younger age group, working in the government sector, less experience in working as a specialist, are more vulnerable. Adequate opportunities should be created for young gastroenterologists to increase their professional skills.

\section{IDDF2020-ABS-0170 THE CLINICAL CHARACTERISTICS AND OUTCOMES OF CROHN'S DISEASE PATIENTS WITH PERIANAL DISEASE ONSET AT PEDIATRIC VERSUS ADULT: A 10-YEAR OBSERVATIONAL STUDY}

${ }^{1}$ Haichao Wang*, ${ }^{1}$ Yalin Wu, ${ }^{2}$ Chen Ye, ${ }^{1}$ Zhanju Liu. ${ }^{1}$ Department of Gastroenterology, The Shanghai Tenth People's Hospital, Tongji University, China; ${ }^{2}$ Medical College of Soochow University, China

\subsection{6/gutjnl-2020-IDDF.106}

Background Perianal disease (PD) is a common complication in Crohn's disease (CD) patients. However, significances of $\mathrm{PD}$, regarding the onset-age, are undefined in perianal Crohn's disease (PCD). We aim to compare PCD patients with pediatric-onset PD (POP) and adult-onset PD (AOP) in terms of their natural history for over 10 years.

Methods Medical records of 293 PCD patients in the Tenth People's Hospital affiliated with Tongji University (Shanghai, China) from January 2008 to December 2017 were reviewed retrospectively. The clinical characteristics and outcomes of patients with POP $(n=84)$ and AOP $(n=209)$ were assessed.

Results Among 293 PCD patients, the mean PD onset-age was 25.9 years. Frequencies of complex perianal fistulas $(71.7 \%$ vs $50.0 \%, p=0.011)$ and infliximab treatment $(33.3 \%$ vs $22.0 \%, p=0.044)$ in POP group were higher than those in AOP group. Medians (interquartile range) diagnostic delay for POP and AOP groups were 12 (2-48) and $24(2.5-60)$ months, respectively. Higher percentage of structuring behavior $(42.1 \%$ vs $27.4 \%, p=0.024)$, current smoking status $(12.9 \%$ vs $4.8 \%, p=0.04)$, and abdominal surgery $(21.1 \%$ vs $4.8 \%, p=0.001)$ were observed in AOP group. Furthermore, structuring behavior was associated with developing PD in adulthood (odds ratio: 2.029, 95\% confidence interval: $1.143-3.604, p=0.016$ ). More recurrence of PD was found in the patients with AOP after withdrawing of infliximab treatment $(38.9 \%$ vs $0 \%, p=0.024)$. The cumulative probabilities of abdominal surgery in POP group were lower than those of in AOP group $(p=0.007)$. Increased use of infliximab was associated with decreased rate of abdominal surgery in patients with AOP $(r=-0.900$, $p=0.037$ ).

Conclusions AOP may indicate more unfavorable outcomes than POP. Those patients with AOP require early and longterm IFX treatment due to their complicated behaviors.

\section{IDDF2020-ABS-0173 EFFICACY AND SAFETY OF THE ENDOSCOPIC RESECTION OF 10- TO 20- MM NON-PEDUNCULATED COLORECTAL POLYPS: A SYSTEMATIC REVIEW AND POOLED ANALYSIS}

${ }^{1}$ Xin Yuan*, 'Zhixin Zhang, ${ }^{2}$ Jiarong Xie, ${ }^{2} Y u$ Zhang, ${ }^{2}$ Hongpeng Lu, ${ }^{2}$ Weihong Wang, ${ }^{2}$ Lei Xu. ${ }^{1}$ College of Medicine, Ningbo University, China; ${ }^{2}$ Department of Gastroenterology, Ningbo First Hospital, China

\subsection{6/gutjpl-2020-IDDF.107}

Background We performed this systematic review and pooled analysis to assess the effectiveness and safety of different endoscopic resection methods for 10 - to 20 -mm non-pedunculated colorectal polyps.

Methods Articles in PubMed, EMBASE, and the Cochrane Library related to the common endoscopic treatment of 10to $20-\mathrm{mm}$ non-pedunculated polyps published as of April 2020 were searched. Primary outcomes were the complete resection rate (CRR) and the en bloc resection rate (EBRR).

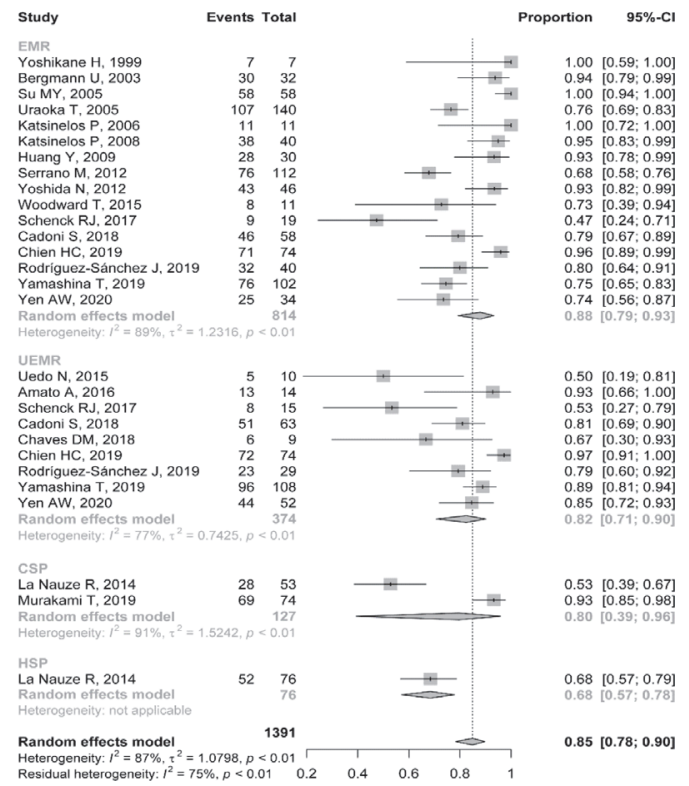

Abstract IDDF2020-ABS-0173 Figure 1 Forest plots reporting the CRRs and EBRRs of different types of resection method 